\title{
Analytical Study of Some Important Generalized Nonlinear Partial Differential Equations
}

\author{
Marwan Alquran, Mahmoud Mohammad, and Ahmad Ababneh \\ Department of Mathematics and Statistics, Jordan University of Science and Technology, Irbid 22110, Jordan \\ Correspondence should be addressed to Marwan Alquran; marwan04@just.edu.jo
}

Received 4 January 2013; Accepted 1 March 2013

Academic Editor: Anjan Biswas

Copyright (C) 2013 Marwan Alquran et al. This is an open access article distributed under the Creative Commons Attribution License, which permits unrestricted use, distribution, and reproduction in any medium, provided the original work is properly cited.

The aim of this paper is to extend the use of homotopy perturbation method (HPM) to study the solutions for some important generalized nonlinear partial differential equations (PDEs) such as Fisher equation with convection term, Sharma-Tasso-Olver (STO) equation, and Fitzhugh-Nagumo (FN) equation.

\section{Introduction}

The main purpose of this paper is to apply the homotopy perturbation method with the help of symbolic computation to obtain approximate solution of the following nonlinear equations.

Fisher equation with convection term serves as a basic model for population dynamics or chemical kinetics in mathematical biology. The solution shows exponential growth counteracted by nonlinear damping (where the damping rate is proportional to $u$ ). Fisher equation has the form

$$
\frac{\partial u}{\partial t}+\alpha u \frac{\partial u}{\partial x}-\frac{\partial^{2} u}{\partial x^{2}}-u(1-u)=0
$$

subject to

$$
u(x, 0)=\frac{1}{2}\left(1-\tanh \left(\frac{x}{4}\right)\right)
$$

Fisher equation was studied by many authors using different methods such as the tanh method [1] and variational iteration method [2].

Sharma-Tasso-Olver (STO) equation is a good example to show the fusion of soliton solutions. It has the form

$$
\frac{\partial u}{\partial t}+\alpha \frac{\partial}{\partial x}\left(u^{3}\right)+\frac{3}{2} \alpha \frac{\partial^{2}}{\partial x^{2}}\left(u^{2}\right)+\alpha \frac{\partial^{3} u}{\partial x^{3}}=0
$$

subject to

$$
u(x, 0)=\sqrt{\frac{1}{\alpha}} \tanh \left(\sqrt{\frac{1}{\alpha}} x\right) .
$$

Hirota's direct method [3] and extended tanh method [4] were used to solve the STO equation.

Fitzhugh-Nagumo (FN) equation is an important nonlinear reaction-diffusion equation and is usually used to model the transmission of nerve impulses; also it is used in circuit theory, biology, and the area of population genetics. FN equation has the form

$$
\frac{\partial u}{\partial t}-\frac{\partial^{2} u}{\partial x^{2}}+u(1-u)(\alpha-u)=0,
$$

subject to

$$
u(x, 0)=\frac{1}{2}\left(1+\tanh \left(\frac{-\sqrt{2}}{4} x\right)\right) .
$$

FN equation was studied by means of improved sinecosine method [5] and Exp-function method [6].

\section{Survey of Homotopy Perturbation Method}

To illustrate the basic ideas of this method, we consider the following nonlinear differential equation:

$$
A(u)-f(r)=0, \quad r \in \Omega,
$$


with the boundary conditions

$$
B\left(u, \frac{\partial u}{\partial \eta}\right)=0, \quad r \in \Gamma
$$

where $A$ is a general differential operator, $B$ is a boundary operator, $f(r)$ is a known analytical function, and $\Gamma$ is the boundary of the domain $\Omega$. The operator $A$ can be divided into two parts, which are $L$ and $N$, where $L$ is the linear, and $N$ is the nonlinear operator. Therefore, (7) can be written as follows:

$$
L(u)+N(u)-f(r)=0
$$

Liao in [7] constructed a homotopy $v(r, p): \Omega \times[0,1] \rightarrow$ $R$, which satisfies

$$
H(v, p)=(1-p)\left[L(v)-L\left(u_{0}\right)\right]+p[A(v)-f(r)]=0,
$$

which is equivalent to

$$
H(v, p)=L(v)-L\left(u_{0}\right)+p\left[L\left(u_{0}\right)+N(v)-f(r)\right]=0,
$$

where $r \in \Omega, p \in[0,1]$, that is, called embedding parameter, $u_{0}$ is an initial approximation solution of (7), which satisfies the boundary conditions. Obviously, from (10) and (11) we obtain

$$
\begin{aligned}
& H(v, 0)=L(v)-L\left(u_{0}\right)=0 \\
& H(v, 1)=A(v)-f(r)=0
\end{aligned}
$$

and the changing process of $p$ from 0 to 1 , is just that of $H(v, p)$ from $L(v)-L\left(u_{0}\right)$ to $A(v)-f(r)$. In topology, this is called deformation, and $L(v)-L\left(u_{0}\right)$ and $A(v)-f(r)$ are called homotopic. Applying the perturbation technique, we can first use the embedding parameter $p$ as a small parameter and assume that the solutions of (10) or (11) can be expressed as a power series in $p$ as follows:

$$
v=\sum_{i=0}^{\infty} p^{i} v_{i}
$$

Setting $p=1$ gives the solution of (7)

$$
u=\lim _{p \rightarrow 1} v=\sum_{i=0}^{\infty} v_{i}
$$

To study the convergence of the method, rewrite (11) in the following form:

$$
L(v)=L\left(u_{0}\right)+p\left[f(r)-L\left(u_{0}\right)-N(v)\right],
$$

applying the inverse operator $L^{-1}$, to both sides of (15), we obtain

$$
v=u_{0}+p\left[L^{-1} f(r)-L^{-1} N(v)-u_{0}\right]
$$

substituting (13) into the right-hand side of (16), we get the following form:

$$
v=u_{0}+p\left[L^{-1} f(r)-\left(L^{-1} N\right)\left(\sum_{i=0}^{\infty} p^{i} v_{i}\right)-u_{0}\right] .
$$

The exact solution may be obtained by using (14)

$$
\begin{aligned}
u & =\lim _{p \rightarrow 1} v \\
& =L^{-1} f(r)-\left(L^{-1} N\right)\left(\sum_{i=0}^{\infty} v_{i}\right) \\
& =L^{-1} f(r)-\sum_{i=0}^{\infty}\left(L^{-1} N\right)\left(v_{i}\right) .
\end{aligned}
$$

The series (14) is convergent for most cases. However the following suggestions have been made by $\mathrm{He}$ [8], to find the convergence rate on nonlinear operator.

(1) The second derivative of $N(v)$ with respect to $v$ must be small because the parameter may be relatively large, that is, $p \rightarrow 1$.

(2) The norm of $L^{-1}(\partial N / \partial v)$ must be smaller than one so that the series converges.

More about this method and illustrative examples, the reader may refer to the following articles [9-17].

\section{Fisher Equation with Convection Term}

Consider the Fisher equation with convection term as follows:

$$
\frac{\partial u}{\partial t}+\alpha u \frac{\partial u}{\partial x}-\frac{\partial^{2} u}{\partial x^{2}}-u(1-u)=0
$$

subject to

$$
u(x, 0)=\frac{1}{2}\left(1-\tanh \left(\frac{x}{4}\right)\right) .
$$

By means of homotopy perturbation technique, the homotopy construction of (19) is

$$
\begin{aligned}
& (1-p)\left(\frac{\partial u}{\partial t}-\frac{\partial u_{0}}{\partial t}\right) \\
& +p\left(\frac{\partial u}{\partial t}+\alpha u \frac{\partial u}{\partial x}-\frac{\partial^{2} u}{\partial x^{2}}-u(1-u)\right)=0
\end{aligned}
$$

or

$$
\frac{\partial u}{\partial t}-\frac{\partial u_{0}}{\partial t}+p\left(\frac{\partial u_{0}}{\partial t}+\alpha u \frac{\partial u}{\partial x}-\frac{\partial^{2} u}{\partial x^{2}}-u(1-u)\right)=0
$$

where the initial approximation $u_{0}=u(x, 0)$. Suppose that the solution of (19) has the form

$$
u=v_{0}+p v_{1}+p^{2} v_{2}+p^{3} v_{3}+\cdots .
$$


Substituting (23) in (22) and comparing the coefficients of identical degrees of $p$, we obtain the following linear equations:

$$
\begin{gathered}
p^{0}: \frac{\partial v_{0}}{\partial t}-\frac{\partial u_{0}}{\partial t}=0 \\
p^{1}: \frac{\partial v_{1}}{\partial t}+\frac{\partial u_{0}}{\partial t}+v_{0}\left(-1+v_{0}+\alpha \frac{\partial v_{0}}{\partial x}\right)-\frac{\partial^{2} v_{0}}{\partial x^{2}}=0 \\
p^{2}: \frac{\partial v_{2}}{\partial t}+v_{1}\left(-1+2 v_{0}+\alpha \frac{\partial v_{0}}{\partial x}\right)+\alpha v_{0} \frac{\partial v_{1}}{\partial x}-\frac{\partial^{2} v_{1}}{\partial x^{2}}=0
\end{gathered}
$$

Solving this system, we obtain the following solutions for $v_{0}, v_{1}, v_{2}$, and so forth:

$$
\begin{gathered}
v_{0}=u(x, 0)=\frac{1}{2}\left(1-\tanh \left(\frac{x}{4}\right)\right), \\
v_{1}=-\int_{0}^{t}\left(\frac{\partial u_{0}}{\partial t}+v_{0}\left(-1+v_{0}+\alpha \frac{\partial v_{0}}{\partial x}\right)-\frac{\partial^{2} v_{0}}{\partial x^{2}}\right) d t \\
=\frac{1}{16} t \operatorname{sech}^{2}\left(\frac{x}{4}\right)\left(4+\alpha-(\alpha-1) \tanh \left(\frac{x}{4}\right)\right), \\
v_{2}=-\int_{0}^{t}\left(v_{1}\left(-1+2 v_{0}+\alpha \frac{\partial v_{0}}{\partial x}\right)+\alpha v_{0} \frac{\partial v_{1}}{\partial x}-\frac{\partial^{2} v_{1}}{\partial x^{2}}\right) d t \\
=\frac{1}{256} t^{2} \operatorname{sech}^{4}\left(\frac{x}{4}\right)((17+2 \alpha(3+\alpha)) \\
\times \sinh \left(\frac{x}{2}\right)-2(\alpha-1) \\
\times(-2(\alpha+3)+(\alpha+4) \\
\times \cosh \left(\frac{x}{2}\right)+(-3+2 \alpha) \\
\left.\left.\times \tanh \left(\frac{x}{4}\right)\right)\right)
\end{gathered}
$$

Considering the first 11 terms of (23), then the approximate solution of (19) by setting $p=1$ is

$$
\begin{aligned}
& u_{\text {app }}(x, t) \\
& =\sum_{i=0}^{10} u_{i}=\frac{1}{2}\left(1-\tanh \left(\frac{x}{4}\right)\right) \\
& \quad+\frac{1}{16} t \operatorname{sech}^{2}\left(\frac{x}{4}\right)\left(4+\alpha-(\alpha-1) \tanh \left(\frac{x}{4}\right)\right)
\end{aligned}
$$

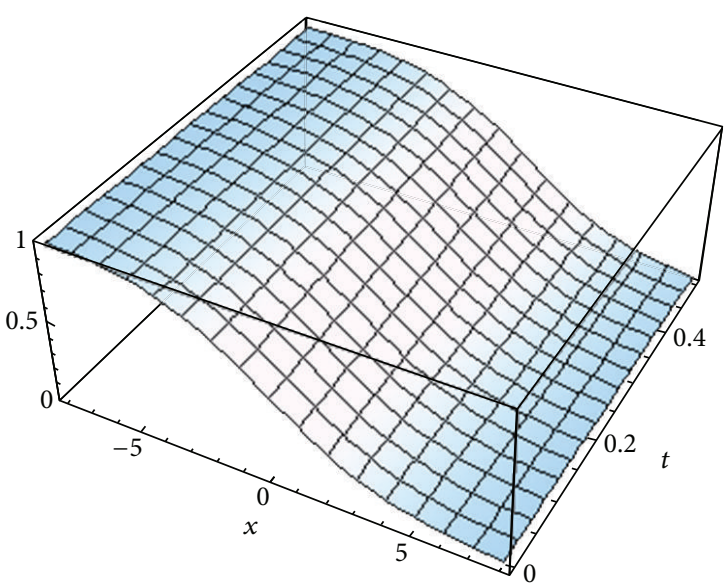

(a)

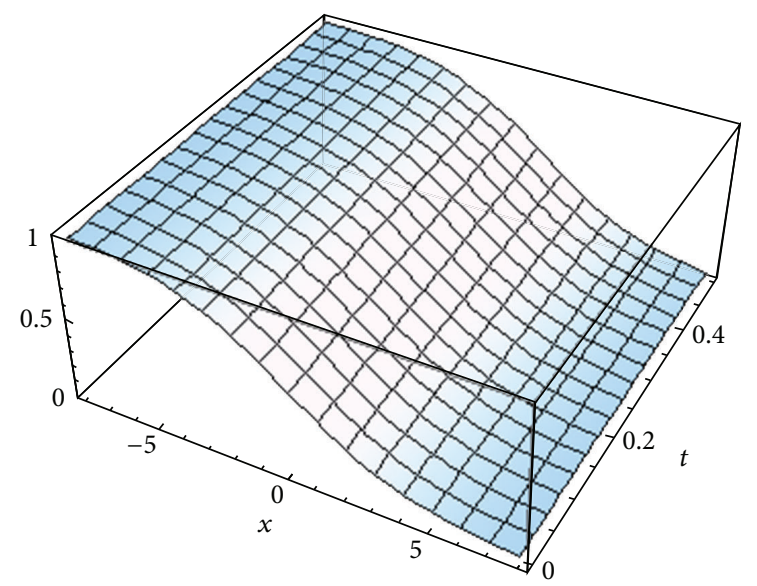

(b)

FIGURE 1: $u(x, t)$ and $u_{\mathrm{app}}(x, t)$ are shown, respectively, from (b) to (a), when $-5 \leq x \leq 5$ and $0 \leq t \leq 0.5$, for Fisher equation, with $n=11, \alpha=1$.

$$
\begin{aligned}
+\frac{1}{256} t^{2} \operatorname{sech}^{4}\left(\frac{x}{4}\right)((17 & +2 \alpha(3+\alpha)) \\
\times & \sinh \left(\frac{x}{2}\right)-2(\alpha-1) \\
\times & (-2(\alpha+3)+(\alpha+4) \\
\times & \cosh \left(\frac{x}{2}\right)+(-3+2 \alpha) \\
\times & \left.\left.\tanh \left(\frac{x}{4}\right)\right)\right)+\cdots
\end{aligned}
$$

The exact solution of (19) is

$$
u(x, t)=\frac{1}{2}\left(1-\tanh \left(\frac{\alpha}{4}\left(x-\left(\frac{\alpha^{2}+4}{2 \alpha}\right) t\right)\right)\right) \text {. }
$$

The behaviors of $u(x, t)$ and $u_{\mathrm{app}}(x, t)$ are shown in Figure 1 and the absolute error is shown in Table 1.

Figures 2 and 3 show that the best choice of $\alpha$ is 1 . 
TABLE 1: Absolute error $\left|u(x, t)-u_{\text {app }}(x, t)\right|$ for Fisher equation, with $n=11$.

\begin{tabular}{lcccc}
\hline$x$ & \multicolumn{3}{c}{$t$} \\
& 0.05 & 0.15 & 0.25 & 0.35 \\
\hline-6 & 0 & $4.4 \times 10^{-16}$ & $1.1 \times 10^{-13}$ & $4.5 \times 10^{-12}$ \\
-3 & 0 & $3.9 \times 10^{-15}$ & $1.1 \times 10^{-12}$ & $4.5 \times 10^{-11}$ \\
0 & 0 & $2.2 \times 10^{-14}$ & $5.9 \times 10^{-12}$ & $2.4 \times 10^{-10}$ \\
3 & $2.8 \times 10^{-17}$ & $3.6 \times 10^{-15}$ & $9.5 \times 10^{-13}$ & $3.7 \times 10^{-11}$ \\
6 & $1.4 \times 10^{-17}$ & $4 \times 10^{-16}$ & $1.2 \times 10^{-13}$ & $5 \times 10^{-12}$ \\
\hline
\end{tabular}

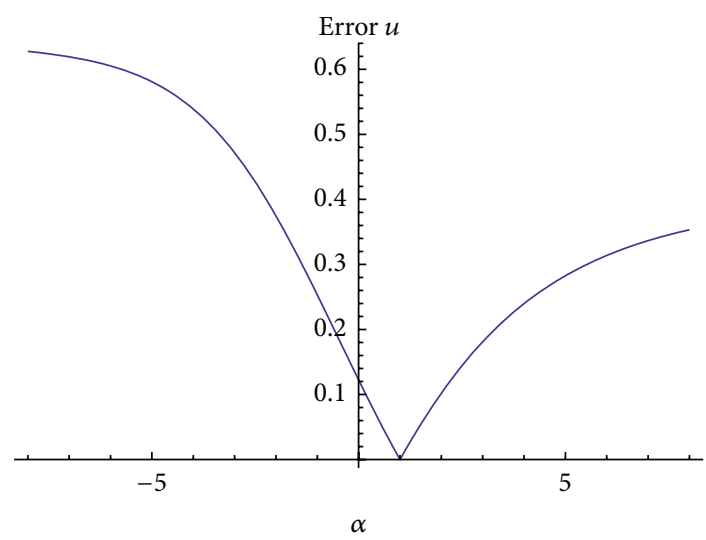

(a)

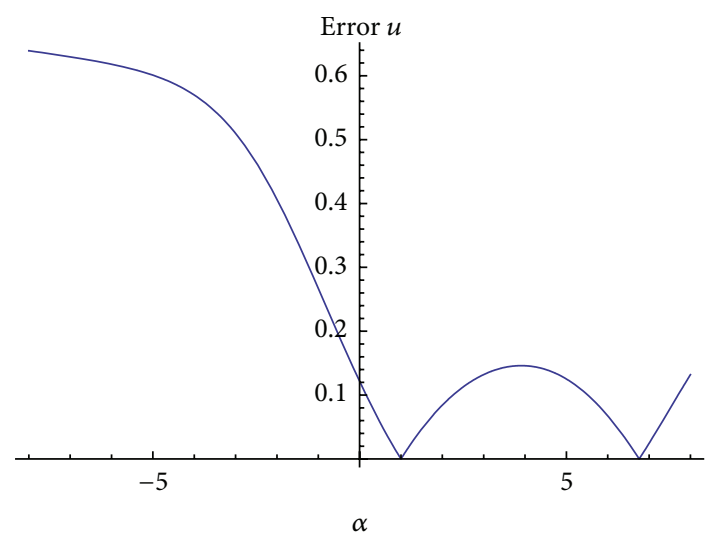

(b)

Figure 2: (a) Absolute error $\left|u(1,0.1)-u_{\text {app }}(1,0.1)\right|$, (b) absolute error $\left|u(1,0.3)-u_{\text {app }}(1,0.3)\right|$ with $n=11$, when $-8 \leq \alpha \leq 8$.

\section{Sharma-Tasso-Olver Equation}

Consider the Sharma-Tasso-Olver equation as follows:

$$
\frac{\partial u}{\partial t}+\alpha \frac{\partial}{\partial x}\left(u^{3}\right)+\frac{3}{2} \alpha \frac{\partial^{2}}{\partial x^{2}}\left(u^{2}\right)+\alpha \frac{\partial^{3} u}{\partial x^{3}}=0
$$

subject to

$$
u(x, 0)=\sqrt{\frac{1}{\alpha}} \tanh \left(\sqrt{\frac{1}{\alpha}} x\right) .
$$

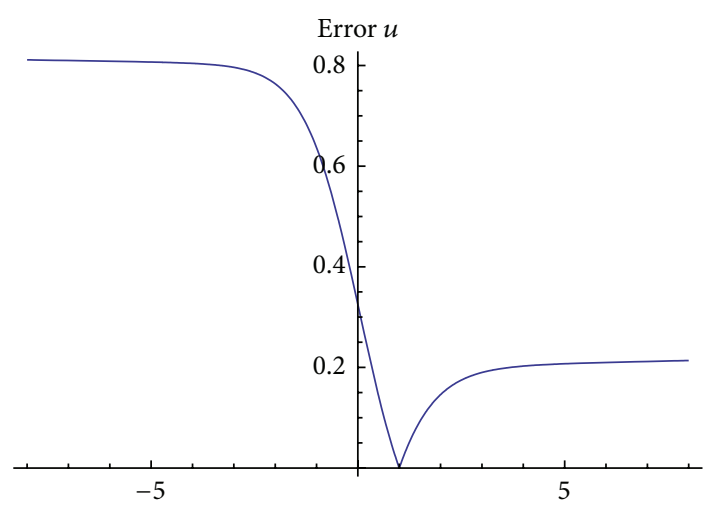

$\alpha$

(a)

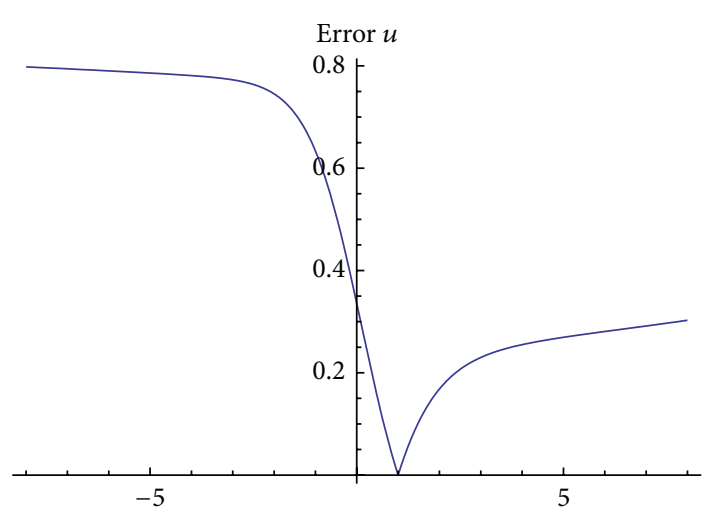

(b)

Figure 3: (a) Absolute error $\left|u(3,0.1)-u_{\text {app }}(3,0.1)\right|$, (b) absolute error $\left|u(3,0.3)-u_{\text {app }}(3,0.3)\right|$ with $n=11$, when $-8 \leq \alpha \leq 8$.

By means of homotopy perturbation technique, the homotopy construction of (28) is

$$
\begin{aligned}
& (1-p)\left(\frac{\partial u}{\partial t}-\frac{\partial u_{0}}{\partial t}\right) \\
& +p\left(\frac{\partial u}{\partial t}+\alpha \frac{\partial}{\partial x}\left(u^{3}\right)+\frac{3}{2} \alpha \frac{\partial^{2}}{\partial x^{2}}\left(u^{2}\right)+\alpha \frac{\partial^{3} u}{\partial x^{3}}\right)=0
\end{aligned}
$$

or

$$
\begin{aligned}
\frac{\partial u}{\partial t}- & \frac{\partial u_{0}}{\partial t} \\
& +p\left(\frac{\partial u_{0}}{\partial t}+\alpha \frac{\partial}{\partial x}\left(u^{3}\right)+\frac{3}{2} \alpha \frac{\partial^{2}}{\partial x^{2}}\left(u^{2}\right)+\alpha \frac{\partial^{3} u}{\partial x^{3}}\right)=0
\end{aligned}
$$

where the initial approximation $u_{0}=u(x, 0)$. Suppose that the solution of (28) has the form

$$
u=v_{0}+p v_{1}+p^{2} v_{2}+p^{3} v_{3}+\cdots
$$


Substituting (32) in (31) and comparing the coefficients of identical degrees of $p$, we obtain the following linear equations:

$$
\begin{gathered}
p^{0}: \frac{\partial v_{0}}{\partial t}-\frac{\partial u_{0}}{\partial t}=0 \\
p^{1}: \frac{\partial v_{1}}{\partial t}+\frac{\partial u_{0}}{\partial t} \\
+\alpha\left(3 \frac{\partial v_{0}}{\partial x}\left(v_{0}^{2}+\frac{\partial v_{0}}{\partial x}\right)+3 v_{0} \frac{\partial^{2} v_{0}}{\partial x^{2}}+\frac{\partial^{3} v_{0}}{\partial x^{3}}\right)=0 \\
p^{2}: \frac{\partial v_{2}}{\partial t}+\alpha\left(3 v_{0}^{2} \frac{\partial v_{1}}{\partial x}+6 \frac{\partial v_{0}}{\partial x} \frac{\partial v_{1}}{\partial x}+3 v_{1} \frac{\partial^{2} v_{0}}{\partial x^{2}}\right. \\
\left.+3 v_{0}\left(2 v_{1} \frac{\partial v_{0}}{\partial x}+\frac{\partial^{2} v_{1}}{\partial x^{2}}\right)+\frac{\partial^{3} v_{1}}{\partial x^{3}}\right)=0
\end{gathered}
$$

Solving this system, we obtain the following solutions for $v_{0}, v_{1}, v_{2}$, and so forth:

$$
\begin{gathered}
v_{0}=u(x, 0)=\sqrt{\frac{1}{\alpha}} \tanh \left(\sqrt{\frac{1}{\alpha}} x\right) \\
v_{1}=-\int_{0}^{t}\left(\frac{\partial u_{0}}{\partial t}+\alpha\left(3 \frac{\partial v_{0}}{\partial x}\left(v_{0}^{2}+\frac{\partial v_{0}}{\partial x}\right)\right.\right. \\
\left.\left.+3 v_{0} \frac{\partial^{2} v_{0}}{\partial x^{2}}+\frac{\partial^{3} v_{0}}{\partial x^{3}}\right)\right) d t \\
=-\frac{1}{\alpha} t \operatorname{sech}^{2}\left(\sqrt{\frac{1}{\alpha}} x\right), \\
+\int_{0}^{t}\left(\alpha \left(3 v_{0}^{2} \frac{\partial v_{1}}{\partial x}+6 \frac{\partial v_{0}}{\partial x} \frac{\partial v_{1}}{\partial x}+3 v_{1} \frac{\partial^{2} v_{0}}{\partial x^{2}}\right.\right. \\
\left.\left.+3 v_{0}\left(2 v_{1} \frac{\partial v_{0}}{\partial x}+\frac{\partial^{2} v_{1}}{\partial x^{2}}\right)+\frac{\partial^{3} v_{1}}{\partial x^{3}}\right)\right) d t \\
=-\alpha^{-3 / 2} t^{2} \operatorname{sech}^{2}\left(\sqrt{\frac{1}{\alpha} x}\right) \tanh \left(\sqrt{\frac{1}{\alpha}} x\right)
\end{gathered}
$$

Considering the first 8 terms of (32), then the approximate solution of (28) by setting $p=1$ is

$$
\begin{aligned}
& u_{\mathrm{app}}(x, t) \\
& =\sum_{i=0}^{7} u_{i}=\sqrt{\frac{1}{\alpha}} \tanh \left(\sqrt{\frac{1}{\alpha}} x\right)-\frac{1}{\alpha} t \operatorname{sech}^{2}\left(\sqrt{\frac{1}{\alpha}} x\right)
\end{aligned}
$$

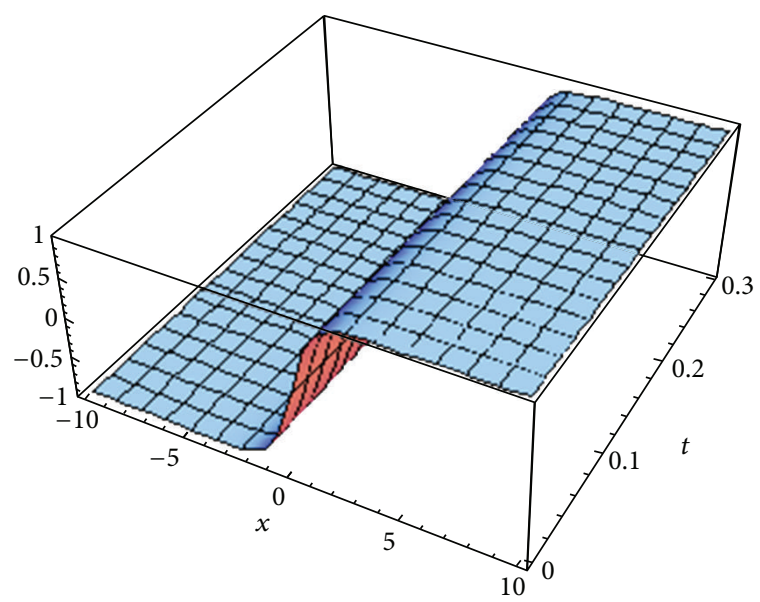

(a)

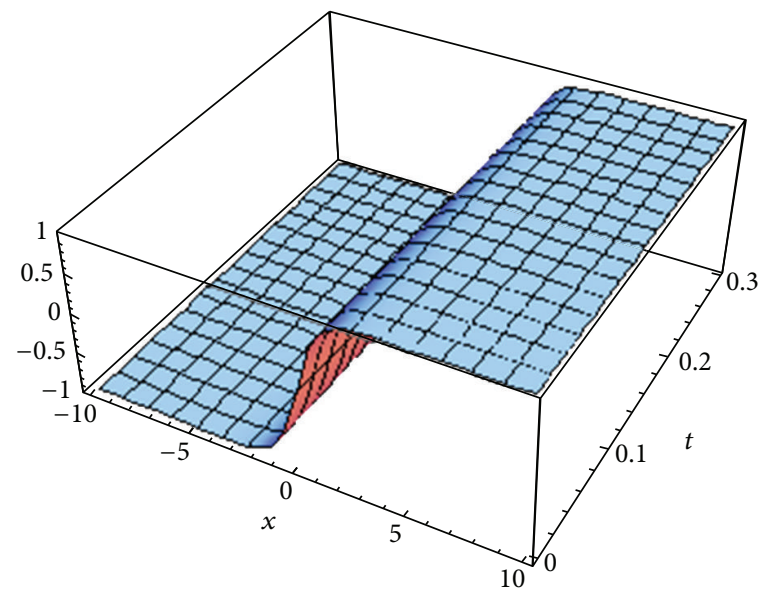

(b)

FIGURE 4: $u(x, t)$ and $u_{\text {app }}(x, t)$ are shown, respectively, from (b) to (a), when $-10 \leq x \leq 10$ and $0 \leq t \leq 0.3$, for STO equation with $n=8, \alpha=1$.

$$
-\alpha^{-3 / 2} t^{2} \operatorname{sech}^{2}\left(\sqrt{\frac{1}{\alpha}} x\right) \tanh \left(\sqrt{\frac{1}{\alpha}} x\right)+\cdots
$$

The exact solution of (28) is

$$
u(x, t)=\sqrt{\frac{1}{\alpha}} \tanh \left(\sqrt{\frac{1}{\alpha}}(x-t)\right) .
$$

4.1. $\alpha=1$. The behavior of $u(x, t)$ and $u_{\text {app }}(x, t)$ is shown in Figure 4 for the regions $-10 \leq x \leq 10$ and $0 \leq t \leq 0.3$. Absolute errors for differences between the exact solution and the 8th order approximate solution given by HPM for $\alpha=1$ are given in Table 2 .

4.2. $\alpha=5$. In this part, we consider $\alpha=5$ and compare it with the above case $\alpha=1$. Figure 5 shows this comparison. One can see that for the case $\alpha=5$ the solution converges rapidly than $\alpha=1$. 
TABLE 2: Absolute error $\left|u(x, t)-u_{\text {app }}(x, t)\right|$ for STO equation with $n=8, \alpha=1$.

\begin{tabular}{lcccc}
\hline$x$ & \multicolumn{3}{c}{$t$} \\
& 0.05 & 0.15 & 0.25 & 0.35 \\
\hline-6 & $1.1 \times 10^{-16}$ & $1.9 \times 10^{-14}$ & $1.1 \times 10^{-12}$ & $1.6 \times 10^{-11}$ \\
-3 & $5.6 \times 10^{-16}$ & $3.3 \times 10^{-12}$ & $2 \times 10^{-10}$ & $2.9 \times 10^{-9}$ \\
0 & $4.3 \times 10^{-14}$ & $8.3 \times 10^{-10}$ & $8.1 \times 10^{-8}$ & $1.6 \times 10^{-6}$ \\
3 & $5.6 \times 10^{-16}$ & $3.2 \times 10^{-12}$ & $1.9 \times 10^{-10}$ & $2.7 \times 10^{-9}$ \\
6 & 0 & $2.1 \times 10^{-14}$ & $1.3 \times 10^{-12}$ & $1.9 \times 10^{-11}$ \\
\hline
\end{tabular}

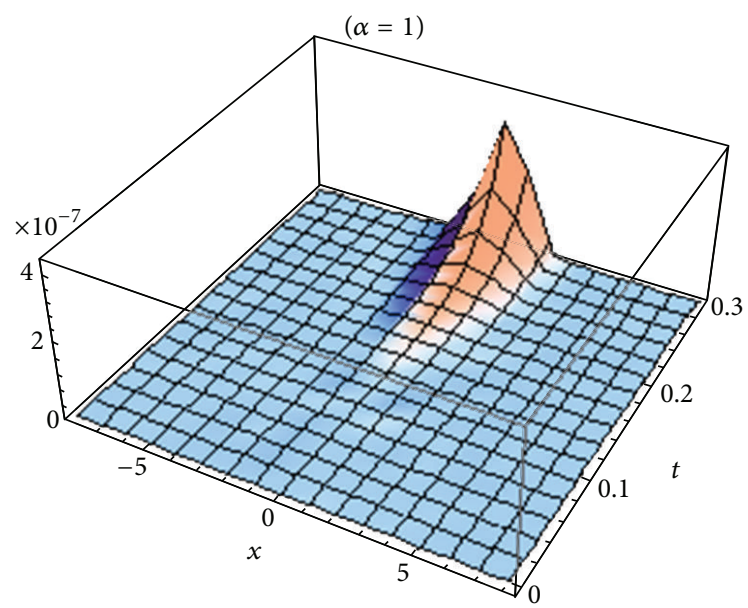

(a)

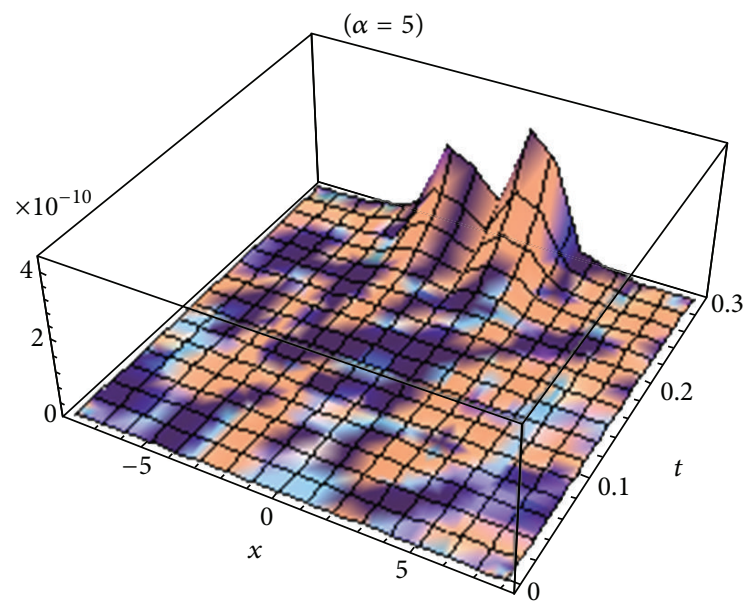

(b)

Figure 5: Absolute errors with respect to $u_{\text {app }}(x, t)$ are shown, respectively, for $\alpha=1$ on (a) and for $\alpha=5$ on (b) when $-8 \leq x \leq 8$ and $0 \leq t \leq 0.3$, for STO equation, with $n=8$.

\section{Fitzhugh-Nagumo Equation}

Consider the Fitzhugh-Nagumo (FN) equation as follows:

$$
\frac{\partial u}{\partial t}-\frac{\partial^{2} u}{\partial x^{2}}+u(1-u)(\alpha-u)=0
$$

subject to

$$
u(x, 0)=\frac{1}{2}\left(1+\tanh \left(\frac{-\sqrt{2}}{4} x\right)\right) .
$$

By means of homotopy perturbation technique, the homotopy construction of (37) is

$$
\begin{aligned}
& (1-p)\left(\frac{\partial u}{\partial t}-\frac{\partial u_{0}}{\partial t}\right) \\
& +p\left(\frac{\partial u}{\partial t}-\frac{\partial^{2} u}{\partial x^{2}}+u(1-u)(\alpha-u)\right)=0
\end{aligned}
$$

or

$$
\frac{\partial u}{\partial t}-\frac{\partial u_{0}}{\partial t}+p\left(\frac{\partial u_{0}}{\partial t}-\frac{\partial^{2} u}{\partial x^{2}}+u(1-u)(\alpha-u)\right)=0
$$

where the initial approximation $u_{0}=u(x, 0)$. Suppose that the solution of (37) has the form

$$
u=v_{0}+p v_{1}+p^{2} v_{2}+p^{3} v_{3}+\cdots .
$$

Substituting (41) in (40) and comparing the coefficients of identical degrees of $p$, we obtain the following linear equations:

$$
\begin{gathered}
p^{0}: \frac{\partial v_{0}}{\partial t}-\frac{\partial u_{0}}{\partial t}=0, \\
p^{1}: \frac{\partial v_{1}}{\partial t}+\frac{\partial u_{0}}{\partial t}+v_{0}\left(\alpha-(1+\alpha) v_{0}+v_{0}^{2}\right)-\frac{\partial^{2} v_{0}}{\partial x^{2}}=0, \\
p^{2}: \frac{\partial v_{2}}{\partial t}+v_{1}\left(\alpha-2(1+\alpha) v_{0}+3 v_{0}^{2}\right)-\frac{\partial^{2} v_{1}}{\partial x^{2}}=0,
\end{gathered}
$$

Solving this system, we obtain the following solutions for $v_{0}, v_{1}, v_{2}$, and so forth:

$$
\begin{gathered}
v_{0}=u(x, 0)=\frac{1}{2}\left(1+\tanh \left(\frac{-\sqrt{2}}{4} x\right)\right), \\
v_{1}=-\int_{0}^{t}\left(\frac{\partial u_{0}}{\partial t}+v_{0}\left(\alpha-(1+\alpha) v_{0}+v_{0}^{2}\right)-\frac{\partial^{2} v_{0}}{\partial x^{2}}\right) d t \\
=\frac{(1-2 \alpha) t}{4(1+\cosh (x / \sqrt{2})}, \\
v_{2}=-\int_{0}^{t}\left(v_{1}\left(\alpha-2(1+\alpha) v_{0}+3 v_{0}^{2}\right)-\frac{\partial^{2} v_{1}}{\partial x^{2}}\right) d t \\
=\frac{(1-2 \alpha)^{2}}{4} t^{2} \operatorname{csch}^{3}\left(\frac{x}{\sqrt{2}}\right) \sinh ^{4}\left(\frac{x}{2 \sqrt{2}}\right),
\end{gathered}
$$




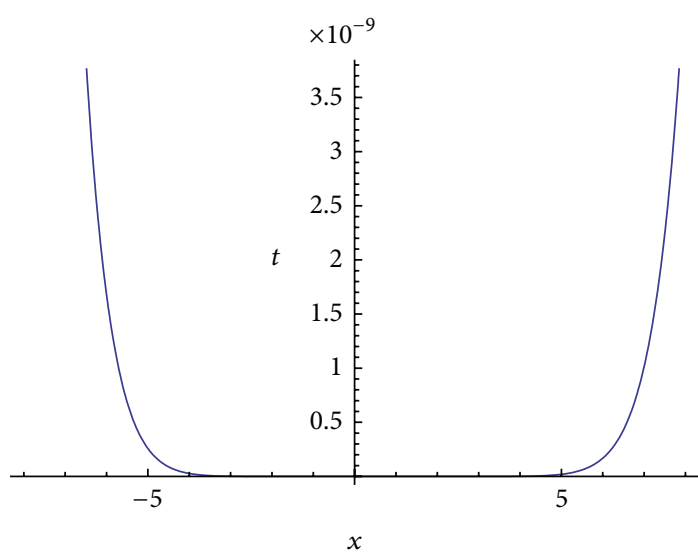

(a)

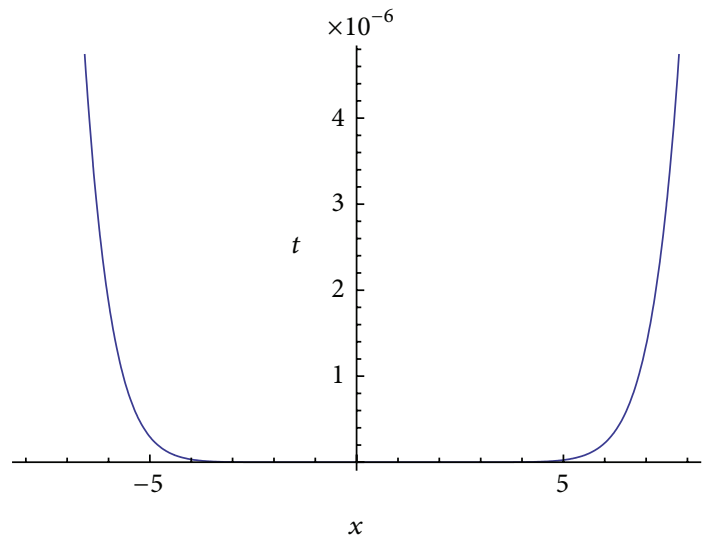

(b)

Figure 6: (a) Absolute error $\left|u(3,0.1)-u_{\text {app }}(3,0.1)\right|$, (b) absolute error $\left|u(7,0.3)-u_{\text {app }}(7,0.3)\right|$, for FN equation, with $n=11$, when $-8 \leq \alpha \leq 8$.

Considering the first 11 terms of (41), then the approximate solution of (37) by setting $p=1$ is

$$
\begin{aligned}
u_{\mathrm{app}}(x, t)= & \sum_{i=0}^{10} u_{i} \\
= & \frac{1}{2}\left(1+\tanh \left(\frac{-\sqrt{2}}{4} x\right)\right) \\
& +\frac{(1-2 \alpha) t}{4(1+\cosh (x / \sqrt{2}))} \\
& +\frac{(1-2 \alpha)^{2}}{4} t^{2} \operatorname{csch}^{3}\left(\frac{x}{\sqrt{2}}\right) \sinh ^{4}\left(\frac{x}{2 \sqrt{2}}\right) \\
& +\cdots .
\end{aligned}
$$

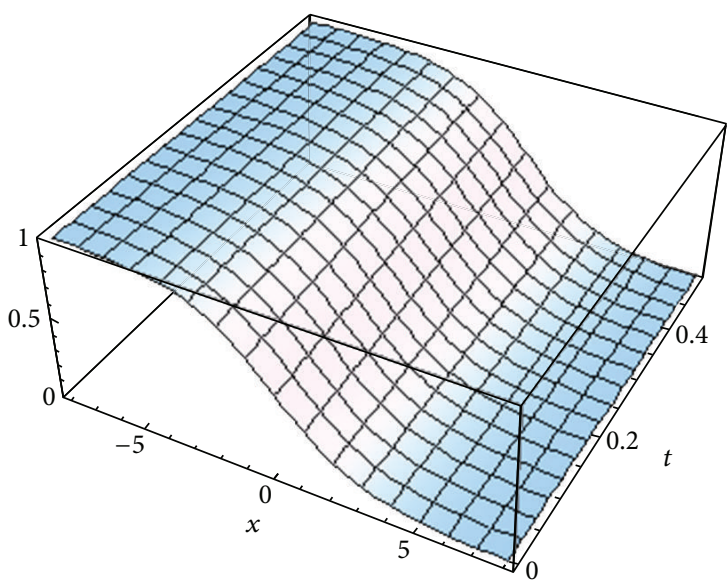

(a)

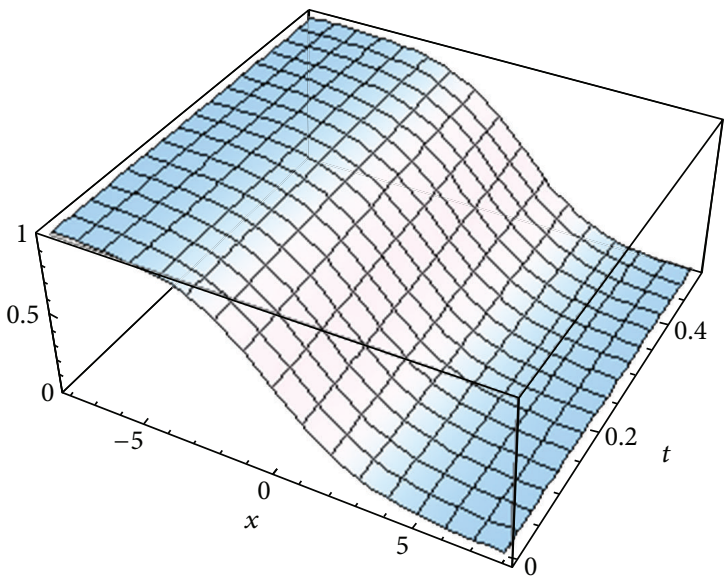

(b)

FIGURE 7: $u(x, t)$ and $u_{\text {app }}(x, t)$ are shown, respectively, from (b) to (a), when $-5 \leq x \leq 5$ and $0 \leq t \leq 0.5$, for NW equation, with $n=11$.

The exact solution of (37) is

$$
u(x, t)=\frac{1}{2}\left(1+\tanh \left(\frac{-\sqrt{2}}{4} x+\left(\frac{1}{4}-\frac{\alpha}{2}\right) t\right)\right) .
$$

Figure 6 shows that the best choice of $\alpha$ is $-5 \leq \alpha \leq 5$, which gives solution that converges rapidly than other choices for $\alpha$. When $\alpha=-1$, the FN equation reduces to the NewellWhitehead (NW) equation, which is an important nonlinear reaction-diffusion equation and is usually used to model the transmission of nerve impulses, also is used in circuit theory, biology, and the area of population genetics as mathematical models.

The behavior of $u(x, t)$ and $u_{\text {app }}(x, t)$ is shown in Figure 7 for the regions $-5 \leq x \leq 5$ and $0 \leq t \leq 0.5$, when $\alpha=-1$.

Absolute errors for differences between the exact solution and the 11th order approximate solution given by HPM for NW equation are given in Table 3. 
TABLE 3: Absolute error $\left|u(x, t)-u_{\text {app }}(x, t)\right|$ for NW equation with $n=11$.

\begin{tabular}{lcccc}
\hline$x$ & \multicolumn{5}{c}{$t$} \\
& 0.05 & 0.15 & 0.25 & 0.35 \\
\hline-6 & $1.1 \times 10^{-16}$ & 0 & $2 \times 10^{-14}$ & $8.5 \times 10^{-13}$ \\
-3 & 0 & $1 \times 10^{-14}$ & $2.7 \times 10^{-12}$ & $1 \times 10^{-10}$ \\
3 & $4.2 \times 10^{-17}$ & $1.2 \times 10^{-14}$ & $3.6 \times 10^{-12}$ & $1.5 \times 10^{-10}$ \\
6 & $1.2 \times 10^{-17}$ & $8 \times 10^{-17}$ & $1.5 \times 10^{-14}$ & $5.4 \times 10^{-13}$ \\
\hline
\end{tabular}

\section{Conclusion}

In this paper, the homotopy perturbation method has been used for finding the approximate solutions of Fisher equation, Sharma-Tasso-Olver equation, and Fitzhugh-Nagumo equation. HPM provides highly accurate numerical solutions for our problems and they also do not require large computer memory and discretization of variable $t$. The approximations are not valid only for small parameters but also for larger ones and the initial approximation can be arbitrarily chosen with unknown constants.

The small size of computations in comparison with the computational size required in characteristics method and the rapid convergence show that the homotopy perturbation method is more reliable and introduces a significant improvement in solving nonlinear partial differential equations.

\section{References}

[1] W. Mafliet, The tanh method in nonlinear wave theory [Habilitation thesis], Department of Physics, University of Antwerp, Antwerp, Belgium, 1994.

[2] M. Matinfar and M. Ghanbari, "Solving the Fisher's equation by means of variational iteration method," International Journal of Contemporary Mathematical Sciences, vol. 4, no. 5-8, pp. 343348, 2009.

[3] S. Wang, X.-Y. Tang, and S.-Y. Lou, "Soliton fission and fusion: burgers equation and Sharma-Tasso-Olver equation," Chaos, Solitons \& Fractals, vol. 21, no. 1, pp. 231-239, 2004.

[4] A.-M. Wazwaz, "New solitons and kinks solutions to the Sharma-Tasso-Olver equation," Applied Mathematics and Computation, vol. 188, no. 2, pp. 1205-1213, 2007.

[5] S. M. Sayed and G. M. Gharib, "Canonical reduction of self-dual Yang-Mills equations to Fitzhugh-Nagumo equation and exact solutions," Chaos, Solitons and Fractals, vol. 39, no. 2, pp. 492498, 2009.

[6] A. Khajeh, A. Yousefi-Koma, M. Vahdat, and M. M. Kabir, "Exact travelling wave solutions for some nonlinear equations arising in biology and engineering," World Applied Sciences Journal, vol. 9, no. 12, pp. 1433-1442, 2010.

[7] S. J. Liao, "An approximate solution technique not depending on small parameters: a special example," International Journal of Non-Linear Mechanics, vol. 30, no. 3, pp. 371-380, 1995.

[8] J.-H. He, "Homotopy perturbation technique," Computer Methods in Applied Mechanics and Engineering, vol. 178, no. 3-4, pp. 257-262, 1999.

[9] J.-H. He, "A coupling method of a homotopy technique and a perturbation technique for non-linear problems," International Journal of Non-Linear Mechanics, vol. 35, no. 1, pp. 37-43, 2000.
[10] J.-H. He, "Homotopy perturbation method: a new nonlinear analytical technique," Applied Mathematics and Computation, vol. 135, no. 1, pp. 73-79, 2003.

[11] J.-H. He, "Recent development of the homotopy perturbation method," Topological Methods in Nonlinear Analysis, vol. 31, no. 2, pp. 205-209, 2008.

[12] J. H. He, "An elementary introduction to recently developed asymptotic methods and nanomechanics in textile engineering," International Journal of Modern Physics B, vol. 22, no. 21, pp. 3487-3578, 2008.

[13] J. Biazar, K. Hosseini, and P. Gholamin, "Homotopy perturbation method for solving KdV and Sawada-Kotera equations," Journal of Applied Mathematics, vol. 6, no. 21, pp. 23-29, 2009.

[14] M. A. Jafari and A. Aminataei, "Improved homotopy perturbation method," International Mathematical Forum, vol. 5, no. 2932, pp. 1567-1579, 2010.

[15] E. Alibeiki and A. Neyrameh, "Application of homotopy perturbation method to nonlinear Drinfeld-Sokolov-Wilson equation," Middle-East Journal of Scientific Research, vol. 10, no. 4, pp. 440-443, 2011.

[16] M. Alquran and M. Mohammad, "Approximate solutions to system of nonlinear partial differential equations using homotopy perturbation method," International Journal of Nonlinear Science, vol. 12, no. 4, pp. 485-497, 2011.

[17] M. A. Abdou and A. A. Soliman, "Modified extended tanhfunction method and its application on nonlinear physical equations," Physics Letters A, vol. 353, no. 6, pp. 487-492, 2006. 


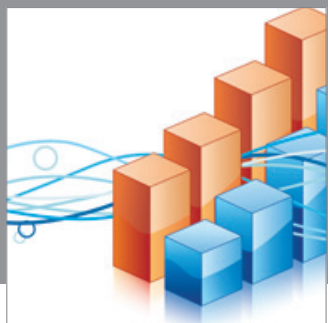

Advances in

Operations Research

mansans

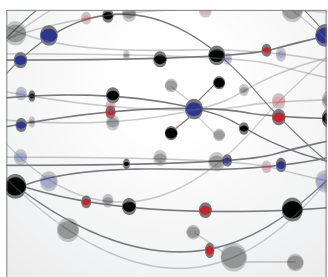

The Scientific World Journal
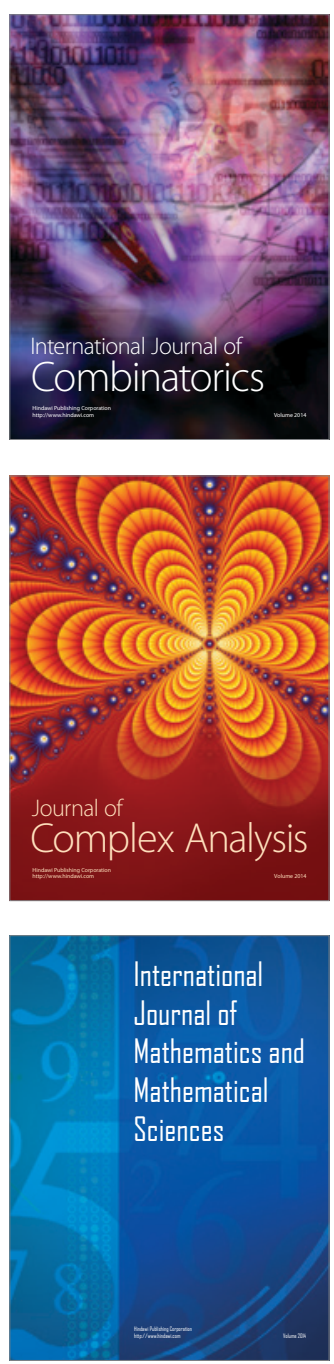
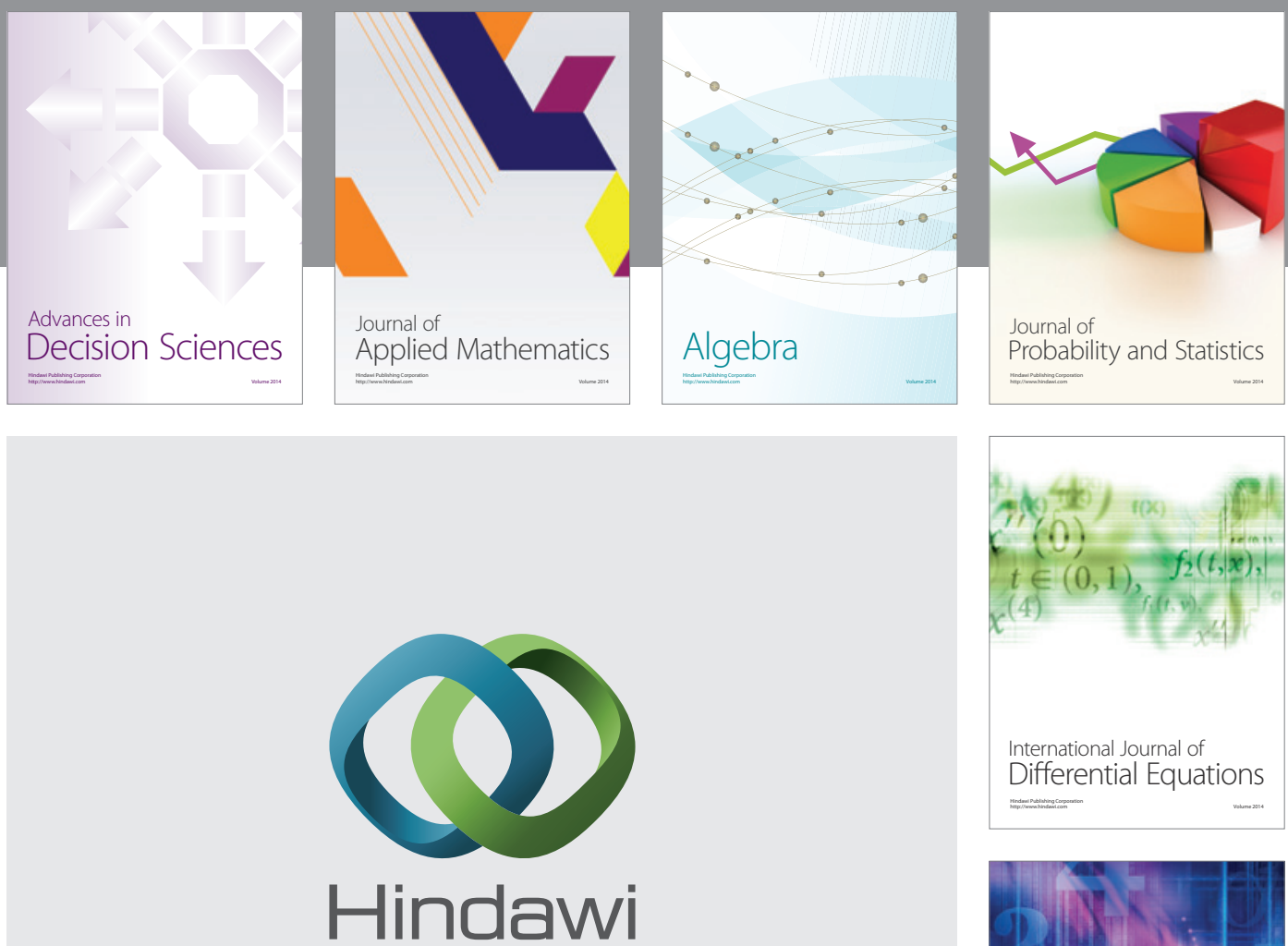

Submit your manuscripts at http://www.hindawi.com
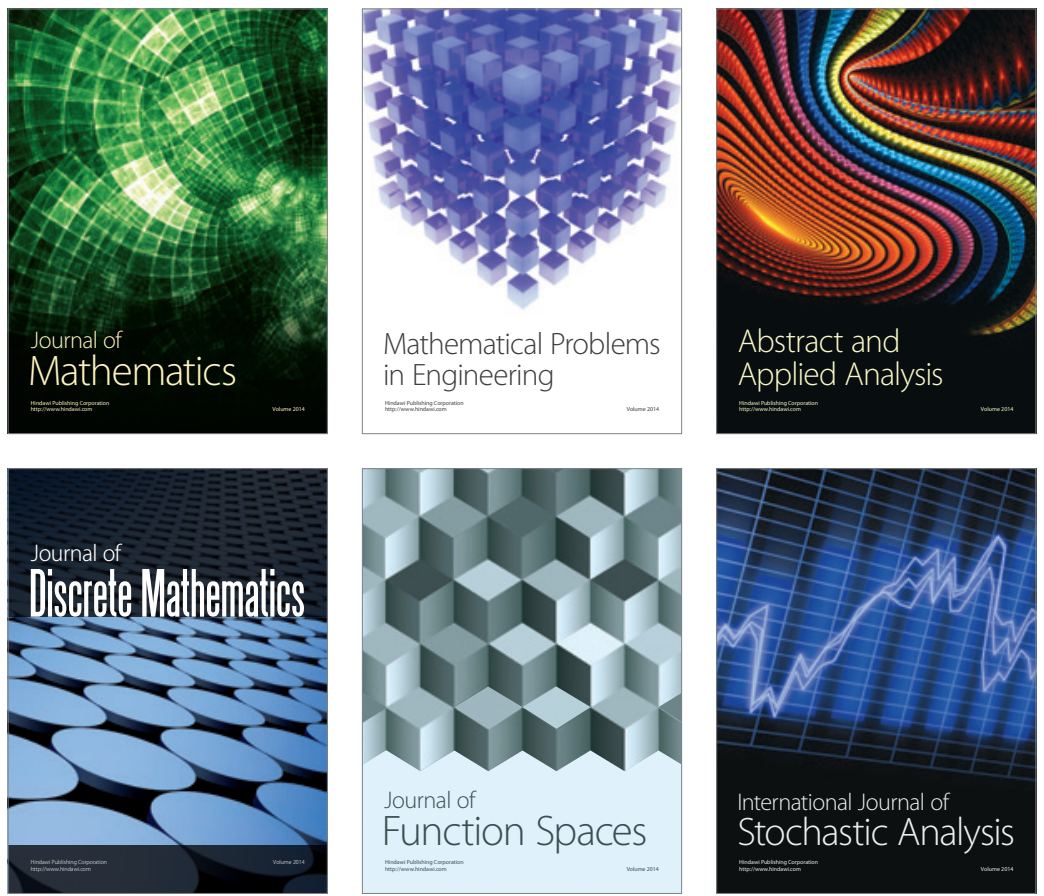

Journal of

Function Spaces

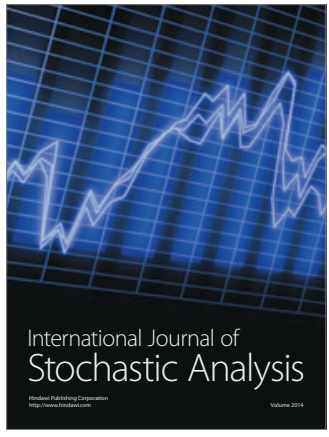

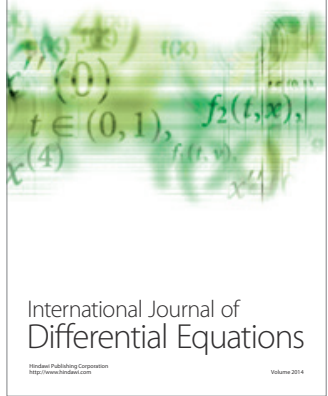
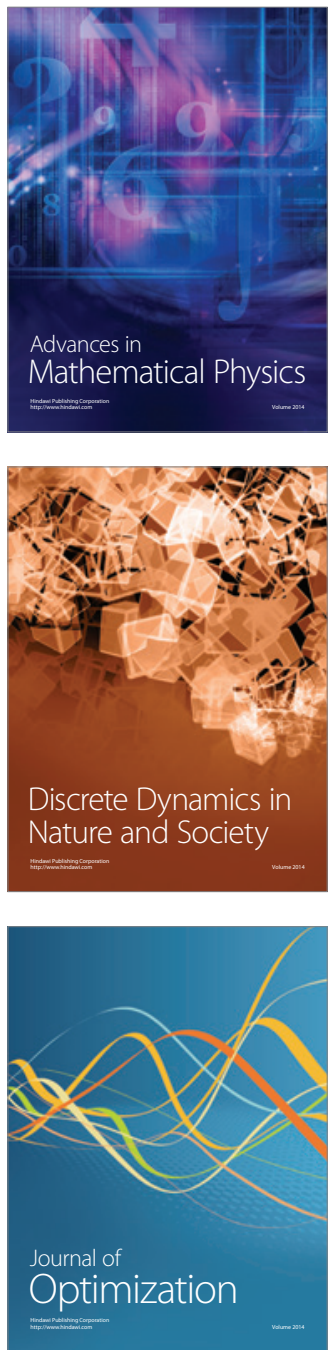